PROCEEDINGS OF THE

AMERICAN MATHEMATICAL SOCIETY

Volume 140, Number 4, April 2012, Pages 1459-1472

S 0002-9939(2011)11050-5

Article electronically published on August 1, 2011

\title{
THE HOMFLY AND DICHROMATIC POLYNOMIALS
}

\author{
XIAN'AN JIN AND FUJI ZHANG
}

(Communicated by Jim Haglund)

\begin{abstract}
In this paper, we first associate a plane graph with an oriented link via replacing each edge of the graph by an alternatingly oriented 2-tangle. Then we establish a relation between the Homfly polynomial of the associated oriented link and the dichromatic polynomial of the plane graph by assigning suitable edge weights. This result extends work of F. Jaeger and L. Traldi.
\end{abstract}

\section{INTRODUCTION}

The paper is inspired by work of F. Jaeger [11 and L. Traldi [22. In [11, F. Jaeger associated an oriented link to a plane graph by replacing each edge of the graph by an oriented clasp as shown in Figure 1 (the highest one). Then he built a relation between the Tutte polynomial 24] of the graph and the Homfly polynomial [10, 19] of the associated oriented link. In [22, L. Traldi introduced a dichromatic polynomial for weighted graphs, which is one of the weighted versions of the Tutte polynomial 2. He, together with T. Przytycka and J. Przytycki, associated a plane graph with an oriented link by replacing each edge by one of four types of oriented clasps as shown in Figure 1. Then he established a relation between the dichromatic polynomial of the plane graph with four different edge weights and the Homfly polynomial of his associated oriented link.

It is natural to consider whether clasps can be generalized to any 2-tangles. We do not know whether arbitrary tangles can be used, but in this paper we prove that arbitrary alternatingly oriented tangles as shown in Figure 2 (right) can be used. To be precise, we first associate a plane graph $G$ with an oriented link $D(G)$ by replacing each edge $e$ of the graph by an alternatingly oriented 2-tangle $T_{e}$. Note that different edges can be replaced by distinct alternatingly oriented 2-tangles. Then we prove that the Homfly polynomial of the associated link can always be obtained, except a factor from the dichromatic polynomial of the weighted plane graph, by assigning suitable weights to the edges. Furthermore, the weight of the edge can be obtained from the Homfly polynomials of the numerator and denominator of the corresponding alternatingly oriented 2-tangle. Our result includes works of [1] and [22] as special cases and thus extends their works. We mention that, however, our result does not provide a description of the Homfly polynomial of an arbitrary link

Received by the editors December 29, 2010.

2000 Mathematics Subject Classification. Primary 57M15, 57M27; Secondary 05CXX.

Key words and phrases. Link, oriented tangle, graph, Homfly polynomial, dichromatic polynomial.

(C)2011 American Mathematical Society Reverts to public domain 28 years from publication 


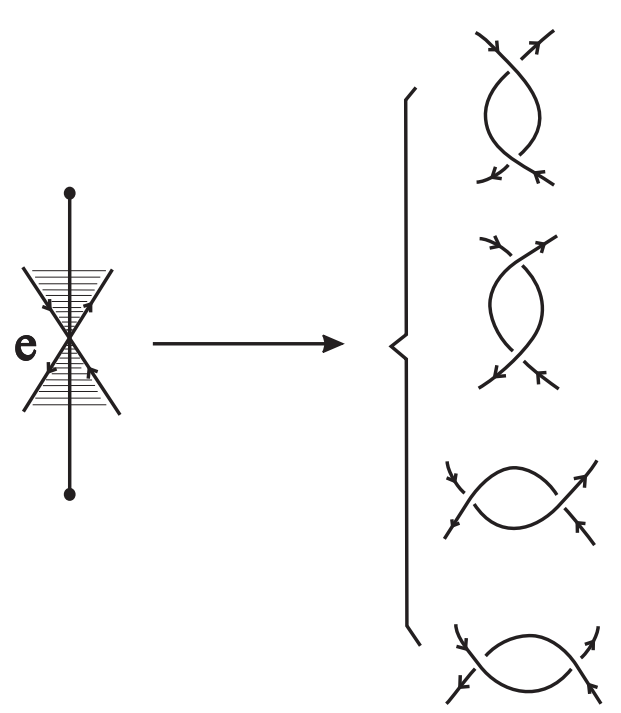

Figure 1. Replacing an edge by four types of oriented clasps; Figure 3 of [22].

diagram using dichromatic polynomials since there exist 2-tangles, say, individual crossings, which are not alternatingly oriented.

The idea of carrying out calculations of knot and link invariants by exhausting the crossings inside each tangle separately is implicit in Conway's 1969 paper [5]. Since then it has been used by researchers who were studying knots and links with the same polynomials. For instance, see [9], 17] and 25]. This idea is also used in our proof of Lemma 4.1. Similarly, the idea of computing Tutte polynomials by analyzing certain particular types of subgraphs or submatroids is not new. It goes back to T. Brylawski [4] and has been further developed by other authors, say, J. G. Oxley and D. J. A. Welsh [18, D. R. Woodall [26], and L. Traldi 23]. The fact that tangle substitutions correspond to 2-sums was noted by W. Schwärzler and D. J. A. Welsh 21]. We mention some fairly recent papers about the computation of Jones polynomials, including [7, 8] and [12.

\section{ThE ORIENTED LINK DIAGRAM ASSOCIATED WITH THE PLANE GRAPH}

The construction of oriented link diagrams associated with plane graphs in this section is essentially the same to those of [1] and 22]. We first need the notation of the medial graph of a plane graph.

A graph is said to be trivial if it is an isolated vertex. The medial graph $M(G)$ of a connected non-trivial plane graph $G$ is a 4-regular plane graph obtained by inserting a vertex on every edge of $G$ and then joining two new vertices by an edge lying in a face of $G$ if the vertices are on adjacent edges of the face. If $G$ is trivial, its medial graph is a simple closed curve surrounding the vertex (strictly, it is not a graph). If $G$ is not connected, its medial graph $M(G)$ is the disjoint union of the medial graphs of its connected components.

Note that the dual of $M(G)$ is bipartite. We can color the faces of the medial graph $M(G)$ white and black in a checkerboard fashion with the unbounded face colored white. Thus the vertices of $G$ correspond to the black faces of $M(G)$ and 
the faces of $G$ correspond to the white faces of $M(G)$. Orienting all edges in the boundary of each black face counterclockwise (or equivalently, orienting edges in the boundary of each white face clockwise), we obtain an edge orientation of $M(G)$.

Let $G$ be a plane graph with oriented medial graph $M(G)$. Replacing each edge $e$ of $G$ with the oriented medial structure on it as shown in Figure 2 (left) by an alternatingly oriented tangle $T_{e}$ as shown in Figure 2 (right), we obtain an oriented link and denote it by $D(G)$. We call a 2-tangle an alternatingly oriented tangle if it has an orientation as shown in Figure 2 (right).

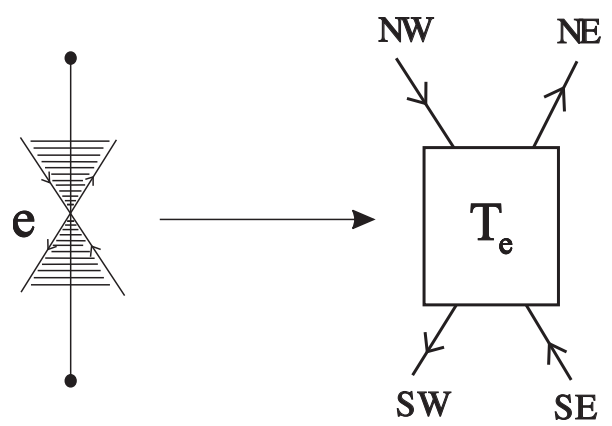

Figure 2. Replacing an edge $e$ by an alternatingly oriented 2tangle $T_{e}$.

We can divide the unoriented 2-tangles into three types according to the connections of their four endpoints inside the tangle boxes. If $\mathrm{NW}$ is connected to NE (resp. SW and SE), the 2-tangle is of type I (resp. II and III). It is clear that a 2-tangle can be alternatingly oriented if and only if it is not of type III. A 2-tangle without circles inside the tangle box is alternatingly oriented if and only if one of its numerator and denominator is a 2-component link and the other is a knot. As the generalization of clasps, the horizontal (resp. vertical) even integer tangle $\overline{[2 n]}$ (resp. $[2 n]$ ) as shown in Figure 3 can be alternatingly oriented. Note that when $n>0$, each crossing of the even integer tangle is positive; when $n<0$, each crossing of the even integer tangle is negative.

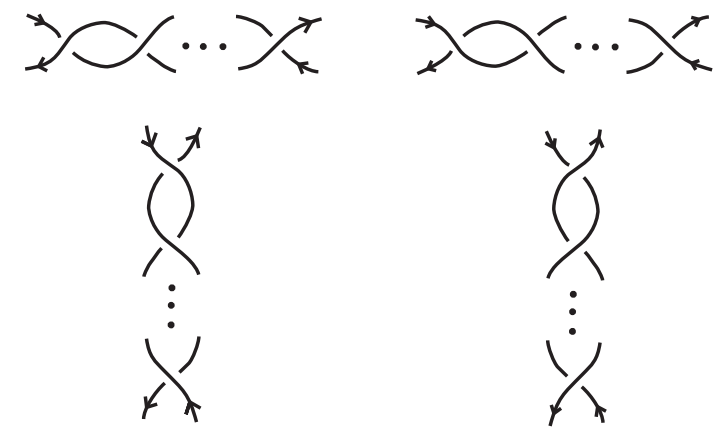

FIGURE 3. Even integer tangles $\overline{[2 n]}$ and $[2 n]$ with $n>0$ (left) and $n<0$ (right). 
Let $T$ be a 2-tangle. By joining with simple arcs the two upper (i.e. NW and $\mathrm{NE}$ ) and the two lower end-points (i.e. SW and SE) of the 2-tangle $T$, we obtain a link called the numerator of $T$, denoted by $N u(T)$; see Figure 4 (left). Joining with simple arcs each pair of the corresponding left end-points (i.e. NW and SW) and right end-points (i.e. $\mathrm{NE}$ and $\mathrm{SE}$ ) of the 2-tangle $T$, we obtain a link called the denominator of $T$, denoted by $D e(T)$; see Figure 4 (right).
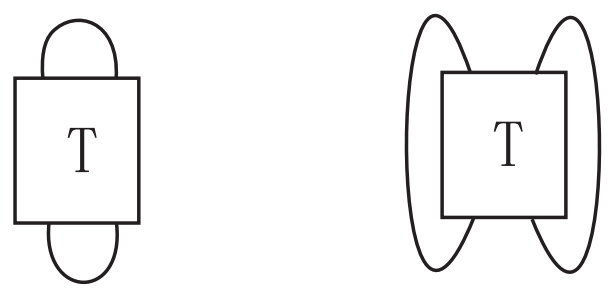

Figure 4. The numerator of $T$ (left) and the denominator of $T$ (right).

\section{Homfly AND DiChromatic POLYNOMIALS}

In this section, we provide some preliminary knowledge on Homfly and dichromatic polynomials.

3.1. The Homfly polynomial. The Homfly polynomial is an invariant of oriented links, introduced in [10] and 19] independently. Now we recall the definition of the Homfly polynomial and some of its basic properties.

Definition 3.1 ([6]). The Homfly polynomial of an oriented link $L$, denoted by $P_{L}(v, z)$, can be defined by the three following axioms:

(1) $P_{L}(v, z)$ is invariant under ambient isotopy of $L$.

(2) If $L$ is the trivial knot, then

$$
P_{L}(v, z)=1 .
$$

(3) Skein relation:

$$
v^{-1} P_{L_{+}}(v, z)-v P_{L_{-}}(v, z)-z P_{L_{0}}(v, z)=0,
$$

where $L_{+}, L_{-}$and $L_{0}$ are link diagrams which are identical except near one crossing where they are as in Figure 5 and are called a skein triple.
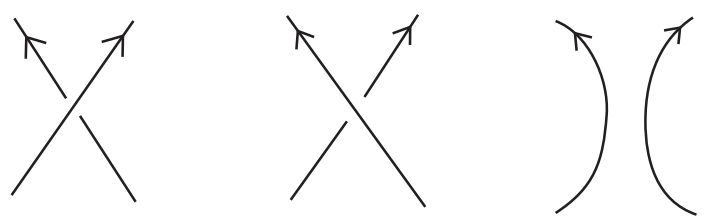

Figure 5. A skein triple: $L_{+}, L_{-}$and $L_{0}$. 
The Alexander [1, Conway [5] and Jones [14 polynomials are all its special cases, i.e.

$$
\begin{aligned}
\Delta_{L}(t) & =P_{L}\left(1, \sqrt{t}-\frac{1}{\sqrt{t}}\right), \\
\nabla_{L}(z) & =P_{L}(1, z), \\
V_{L}(t) & =P_{L}\left(t, \sqrt{t}-\frac{1}{\sqrt{t}}\right) .
\end{aligned}
$$

The following basic property of the Homfly polynomial will be used. If $L_{1} \sqcup L_{2}$ is the disjoint union of two oriented links $L_{1}$ and $L_{2}$, then [6]

$$
P_{L_{1} \sqcup L_{2}}(v, z)=\frac{v^{-1}-v}{z} P_{L_{1}}(v, z) P_{L_{2}}(v, z) .
$$

In particular,

$$
P_{\bigcirc \sqcup L}(v, z)=\frac{v^{-1}-v}{z} P_{L}(v, z),
$$

where $\bigcirc$ is a trivial knot.

3.2. The dichromatic polynomial of weighted graphs. A weighted graph is a graph $G$ together with a function $w$ mapping $E(G)$ into some commutative ring $R$ with unity 1 . If $e$ is an edge of $G$, then $w(e)$ is the weight of the edge $e$.

The dichromatic polynomial for weighted graphs was introduced by L. Traldi in [22], which is the generalization of the Tutte polynomial for graphs constructed by Tutte [24] and the Tutte polynomial for signed graphs introduced by L. H. Kauffman [15].

Let $G=(V, E)$ be a graph. We denote by $k(G)$ the number of connected components of $G$. We use $r(G)=|V|-k(G)$ and $n(G)=|E|-|V|+k(G)$ to denote the rank and nullity of the graph $G$, respectively.

Definition 3.2. The dichromatic polynomial $Q_{G}(t, z)$ of a weighted graph $G$ is defined as

$$
Q_{G}(t, z)=\sum_{F \subset E(G)}\left(\prod_{f \in F} w(f)\right) t^{k\langle F\rangle} z^{n\langle F\rangle},
$$

where $k\langle F\rangle$ and $n\langle F\rangle$ are the number of connected components and the nullity of the spanning subgraph $\langle F\rangle$, induced by $F$, of $G$, respectively.

When $w(e)=1$ for each edge $e$ of $G, Q_{G}(t, z)$ reduces to a variant of the Tutte polynomial, called the Whitney-Tutte polynomial in [2. We should point out that despite the fact that the dichromatic polynomial is defined to be a polynomial in $t$ and $z$, we do not require that $t$ and $z$ be indeterminates; indeed, it is often convenient to treat $t$ and $z$ as elements of $R$.

Let $e$ be an edge of the graph $G$. We shall use $G-e$ and $G / e$ to denote the graphs obtained from $G$ by deleting the edge $e$ and contracting the edge $e$ (i.e. deleting the edge $e$ first and then identifying its two endvertices), respectively. When $e$ is a loop, $G-e=G / e$. The following recursive relations hold, which may be taken as an alternative definition of the polynomial; see [22.

(1) If $G$ is an edgeless graph with $n \geq 1$ vertices,

$$
Q_{G}=t^{n} \text {. }
$$


(2) If $e$ is a loop of $G$, then

$$
Q_{G}=(1+w(e) z) Q_{G-e} .
$$

If $e$ is not a loop of $G$, then

$$
Q_{G}=Q_{G-e}+w(e) Q_{G / e} .
$$

In the next section we shall study the possibility of computing the Homfly polynomials of $D(G)$ via the dichromatic polynomial of weighted graph $G$ and establish a relation between the two.

\section{The Relation Between the two}

Let $G$ be a plane graph and $D(G)$ be its associated oriented link. In this section we shall study the relation between the Homfly polynomial of $D(G)$ and the dichromatic polynomial of weighted $G$.

Let $G$ be a plane graph and $D(G)$ be its associated oriented link diagram. Let $e$ be an edge of $G$. Then $G-e$ and $G / e$ are also plane graphs. We use $D(G-e)$ and $D(G / e)$ to denote the oriented link diagrams associated with $G-e$ and $G / e$ with the oriented tangles to replace edges in $E-\{e\}$ unchanged, respectively.

Lemma 4.1. Let $e$ be an edge of the plane graph $G$. Then

(1) When e is a loop,

$$
P_{D(G)}=L(e ; v, z) P_{D(G-e)},
$$

where $L(e ; v, z)$ is a Laurent polynomial in $v$ and $z$, and depend on the edge $e$.

(2) When e is not a loop,

$$
P_{D(G)}=M(e ; v, z) P_{D(G-e)}+N(e ; v, z) P_{D(G / e)},
$$

where $M(e ; v, z)$ and $N(e ; v, z)$ are Laurent polynomials in $x, y$ and $z$, and depend on the edge $e$.

Proof. (1) $e$ is a loop.

Note that in this case $D(G)$ is the connected sum of $D(G-e)$ and $D e\left(T_{e}\right)$; we have

$$
P_{D(G)}=P_{D e\left(T_{e}\right)} P_{D(G-e)} .
$$

Thus, the lemma holds.

(2) $e$ is not a loop.

We shall prove the lemma by induction on the number $c\left(T_{e}\right)$ of the crossings of the tangle $T_{e}$. If $c\left(T_{e}\right)=0$, then

(1) If the end $N W$ of the tangle $T_{e}$ is connected, via the tangle box, to the end $N E$ and the end $S E$ is connected to the end $S W$, then $D(G)=D(G-e)$ except possible disjoint simple circles. The lemma holds.

(2) If $c\left(T_{e}\right)=0$ and the end $N W$ of the tangle $T_{e}$ is connected, via the tangle box, to the end $S W$ and the end $S E$ is connected to the end $N E$, then $D(G)=D(G / e)$ except possible disjoint simple circles. The lemma holds.

Suppose that the lemma holds for $c\left(T_{e}\right)<l$. Now we consider the tangle $T_{e}$ with $c\left(T_{e}\right)=l$. There are two cases: 
(a) The end $N W$ of the tangle $T_{e}$ is connected, via the tangle box, to the end $N E$, and the end $S E$ is connected to the end $S W$.

By switching a sequence of crossings of $T_{e}$, we can separate $T_{e}$ to a tangle $T_{e}^{\prime}$, which is the disjoint union of the $\operatorname{arc} \widehat{N W N E}, \widehat{S E S W}$ and (possibly) the remaining link diagram $L_{e}$. Let $D^{\prime}(G)$ be the link diagram obtained from $D(G)$ by substituting $T_{e}$ with $T_{e}^{\prime}$. Then, by Eq. (3.3), we have

$$
P_{D^{\prime}(G)}=\frac{v^{-1}-v}{z} P_{L_{e}} P_{D(G-e)} .
$$

If $L_{e}$ does not exist, then $D^{\prime}(G)=D(G-e)$.

Thus, by applying the skein relation successively, we can convert the Homfly polynomial of $D(G)$ to the combination of those of $D^{\prime}(G)$ and some $D^{\prime \prime}(G)$ 's obtained from $D(G)$ by changing $T_{e}$ to $T_{e}^{\prime \prime}$ with $c\left(T_{e}^{\prime \prime}\right)<l$.

In Figure 6, we give an example and its process of the conversion.

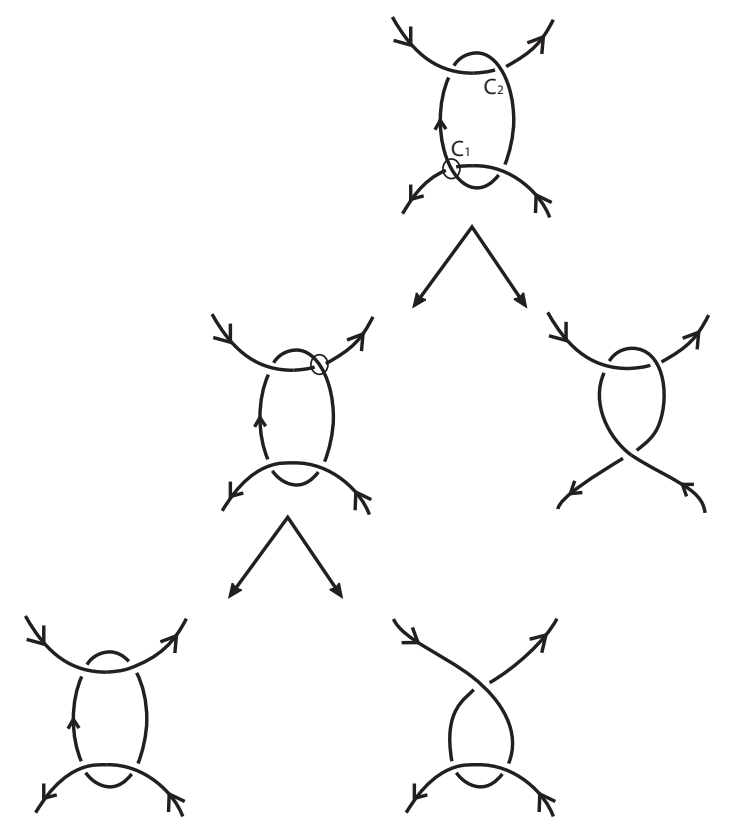

Figure 6 . The process of the conversion of an example.

(b) The end $N W$ of the tangle $T_{e}$ is connected, via the tangle box, to the end $S W$, and the end $S E$ is connected to the end $N E$.

By switching a sequence of crossings of $T_{e}$, we can separate $T_{e}$ to a tangle $T_{e}^{\prime}$ which is the disjoint union of the arc $\widehat{N W S W}, \widehat{S E N E}$ and (possibly) the remaining link diagram $L_{e}$. Let $D^{\prime}(G)$ be the link diagram obtained from $D(G)$ by substituting $T_{e}$ with $T_{e}^{\prime}$. Then, by Eq. (3.3), we have

$$
P_{D^{\prime}(G)}=\frac{v^{-1}-v}{z} P_{L_{e}} P_{D(G / e)} .
$$

If $L_{e}$ does not exist, then $D^{\prime}(G)=D(G / e)$. 
Thus, by applying the skein relation successively, we can convert the Homfly polynomial of $D(G)$ to the combination of those of $D^{\prime}(G)$ and some $D^{\prime \prime}(G)$ 's obtained from $D(G)$ by changing $T_{e}$ to $T_{e}^{\prime \prime}$ with $c\left(T_{e}^{\prime \prime}\right)<l$.

By the induction hypothesis, the lemma holds.

Theorem 4.2. Let $G$ be a plane graph and $D(G)$ be its associated link diagram. Then $P_{D(G)}(v, z)$ can be written as

$$
\left(\frac{z}{v^{-1}-v}\right)\left(\prod_{e \in E(G)} \mu(e)\right) Q_{G}(\alpha(v, z), \beta(v, z))
$$

if and only if $\frac{L(e ; v, z)-M(e ; v, z)}{N(e ; v, z)}$ is independent of the edge e. Furthermore, $\mu(e)=$ $M(e ; v, z), \alpha(v, z)=\frac{v^{-1}-v}{z}, \beta(v, z)=\frac{L(e ; v, z)-M(e ; v, z)}{N(e ; v, z)}$ and the weight $w(e)$ of edge e of $G$ is $\frac{N(e ; v, z)}{M(e ; v, z)}$.

Proof. (1) The sufficiency $(\Leftarrow)$. Suppose that $P_{D(G)}(v, z)$ is the Homfly polynomial of $D(G)$ and $\frac{L(e ; v, z)-M(e ; v, z)}{N(e ; v, z)}$ does not depend on the edge $e$. Let

$$
\tilde{P}_{D(G)}(v, z)=\left(\frac{v^{-1}-v}{z}\right) \frac{P_{D(G)}(v, z)}{\prod_{e \in E(G)} M(e ; v, z)} .
$$

By Eqs. (4.1) and (4.2), we have

$$
\tilde{P}_{D(G)}=\frac{L(e ; v, z)}{M(e ; v, z)} \tilde{P}_{D(G-e)}
$$

and

$$
\tilde{P}_{D(G)}=\tilde{P}_{D(G-e)}+\frac{N(e ; v, z)}{M(e ; v, z)} \tilde{P}_{D(G / e)} .
$$

When $G$ is an edgeless graph with $n \geq 1$ vertices, $D(G)$ is the trivial link with $n$ components. We have

$$
\tilde{P}_{D(G)}=\left(\frac{v^{-1}-v}{z}\right)^{n} .
$$

Comparing (4.3)-(4.5) with (3.5)-(3.7), if $\frac{L(e ; v, z)-M(e ; v, z)}{N(e ; v, z)}$ does not depend on the edge $e$, we obtain

$$
\tilde{P}_{D(G)}=Q_{G}\left(\frac{v^{-1}-v}{z}, \frac{L(e ; v, z)-M(e ; v, z)}{N(e ; v, z)}\right),
$$

where the weight $w(e)$ of edge $e$ of $G$ is $\frac{N(e ; v, z)}{M(e ; v, z)}$. Hence, $P_{D(G)}(v, z)$ can be written as $\left(\frac{z}{v^{-1}-v}\right)\left(\prod_{e \in E(G)} \mu(e)\right) Q_{G}(\alpha(v, z), \beta(v, z))$.

$(2)$ The necessity $(\Rightarrow)$. Let

$$
\tilde{Q}_{G}(v, z)=\left(\frac{z}{v^{-1}-v}\right)\left(\prod_{e \in E(G)} \mu(e)\right) Q_{G}(\alpha(v, z), \beta(v, z)) .
$$

Then

(1) If $G$ is an edgeless graph with $n \geq 1$ vertices,

$$
\tilde{Q}_{G}=\left(\frac{z}{v^{-1}-v}\right) \alpha(v, z)^{n} .
$$


(2) If $e$ is a loop of $G$, then

$$
\tilde{Q}_{G}=\mu(e)(1+w(e) \beta(v, z)) \tilde{Q}_{G-e} .
$$

If $e$ is not a loop of $G$, then

$$
\tilde{Q}_{G}=\mu(e) \tilde{Q}_{G-e}+\mu(e) w(e) \tilde{Q}_{G / e} .
$$

Note that if $G$ is the empty graph with $n$ vertices, then $P_{D(G)}=\left(\frac{v^{-1}-v}{z}\right)^{n-1}$. By Eq. (4.6) we obtain $\alpha(v, z)=\frac{v^{-1}-v}{z}$. Comparing Eqs. (4.7)-(4.8) with (4.1)-(4.2), we obtain $\mu(e)=M(e ; v, z), w(e)=\frac{N(e ; v, z)}{M(e ; v, z)}$ and $\beta(v, z)=\frac{L(e ; v, z)-M(e ; v, z)}{N(e ; v, z)}$, which does not depend on the edge $e$.

Now we consider some special cases.

Let the weight of the edge replaced by the vertical (resp. horizontal) integer tangle $\left[2 n_{i}\right]$ (resp. $\overline{\left[2 n_{i}\right]}$ ) be $\frac{v^{-1}-v}{z} \frac{v^{2 n_{i}}}{1-v^{2 n_{i}}}$ (resp. $\frac{z}{v^{-1}-v} \frac{1-v^{2 n_{i}}}{v^{2 n_{i}}}$ ).

Theorem 4.3. Let $G$ be a plane graph. Let $D_{r}(G)$ be the oriented link diagram obtained from $G$ by replacing the edges by even integer tangles. If the edges of $G$ are assigned weights as described above, then

$$
P_{D_{r}(G)}(v, z)=\left(\frac{z}{v^{-1}-v}\right)\left(\prod_{e \in E(G)} \nu(e)\right) Q_{G}\left(\frac{v^{-1}-v}{z}, \frac{v^{-1}-v}{z}\right),
$$

where $\nu(e)$ is $\frac{z}{v^{-1}-v}\left(1-v^{2 n}\right)$ (resp. $v^{2 n}$ ) if $e$ is replaced by the vertical (resp. horizontal) integer tangle $[2 n]$ (resp. $\overline{[2 n]}$ ).

Proof. When $T_{e}=\left[2 n_{e}\right]$, we have

$$
\begin{aligned}
L(e ; v, z) & =\frac{z}{v^{-1}-v}+\left(\frac{v^{-1}-v}{z}-\frac{z}{v^{-1}-v}\right) v^{2 n_{e}}, \\
M(e ; v, z) & =\frac{z}{v^{-1}-v}\left(1-v^{2 n_{e}}\right), \\
N(e ; v, z) & =v^{2 n_{e}} .
\end{aligned}
$$

Hence, $\beta(v, z)=\frac{v^{-1}-v}{z}$.

When $T_{e}=\overline{\left[2 n_{e}\right]}$, we have

$$
\begin{aligned}
L(e ; v, z) & =1 \\
M(e ; v, z) & =v^{2 n_{e}}, \\
N(e ; v, z) & =\frac{z}{v^{-1}-v}\left(1-v^{2 n_{e}}\right) .
\end{aligned}
$$

Hence, $\beta(v, z)=\frac{v^{-1}-v}{z}$.

This result generalizes Theorem 5 in [22] and Theorem 4.2 in [13] and partly results in [16]. The following are two extreme cases of Theorem 4.3.

Corollary 4.4 ([13]). Let $G$ be a plane graph with edge set $E(G)=\left\{e_{1}, e_{2}, \cdots, e_{m}\right\}$. Let $D_{c}(G)$ be the associated link diagram obtained from $G$ by replacing the edge $e_{i}$ by the vertical integer tangle $\left[2 n_{i}\right]$. If we let the weight of $e_{i}$ be $\frac{v^{-1}-v}{z} \frac{v^{2 n_{i}}}{1-v^{2 n_{i}}}$, then

$$
P_{D_{c}(G)}(v, z)=\left(\frac{z}{v^{-1}-v}\right)^{m+1}\left(\prod_{i=1}^{m}\left(1-v^{2 n_{i}}\right)\right) Q_{G}\left(\frac{v^{-1}-v}{z}, \frac{v^{-1}-v}{z}\right) .
$$


Corollary 4.5. Let $G$ be a plane graph with edge set $E(G)=\left\{e_{1}, e_{2}, \cdots, e_{m}\right\}$. Let $D_{s}(G)$ be the associated link diagram obtained from $G$ by replacing the edge $e_{i}$ by the horizontal integer tangle $\overline{\left[2 n_{i}\right]}$. If we let the weight of $e_{i}$ be $\frac{z}{v^{-1}-v} \frac{1-v^{2 n_{i}}}{v^{2 n_{i}}}$, then

$$
P_{D_{s}(G)}(v, z)=\left(\frac{z}{v^{-1}-v}\right)\left(\prod_{i=1}^{m} v^{2 n_{i}}\right) Q_{G}\left(\frac{v^{-1}-v}{z}, \frac{v^{-1}-v}{z}\right) .
$$

The following special case of Corollaries 4.4 and 4.5 may be of interest and extends Corollary 3 of [1].

Corollary 4.6. Let $G$ be a connected plane graph. Let $\nabla_{L}(z)$ be the Conway polynomial of the oriented link $L$. Then

$$
\begin{aligned}
& \nabla_{D_{c}(G)}(z)=z^{n(G)} \sum_{T} \prod_{e \notin T} n_{e}, \\
& \nabla_{D_{s}(G)}(z)=z^{r(G)} \sum_{T} \prod_{e \in T} n_{e},
\end{aligned}
$$

where the sum is over all spanning trees $T$ of $G$.

Proof. It is well known that the Conway polynomial is the special case $v=1$ of the Homfly polynomial.

(1) The case of $D_{c}(G)$.

In this case $w(e)=\frac{1}{n_{e} z}$ and $\nu(e)=n_{e} z$. Hence,

$$
\begin{aligned}
\nabla_{D_{c}(G)}(z) & =\left.\left(\frac{z}{v^{-1}-v}\right)\left(\prod_{e \in E(G)} \nu(e)\right) Q_{G}\left(\frac{v^{-1}-v}{z}, \frac{v^{-1}-v}{z}\right)\right|_{v=1} \\
& =\left(\prod_{e \in E(G)} \nu(e)\right) \sum_{F \subset E(G)}\left(\prod_{f \in F} w(f)\right)\left(\frac{v^{-1}-v}{z}\right)^{k\langle F\rangle-1}\left(\frac{v^{-1}-v}{z}\right)^{n\langle F\rangle} \\
& =\left(\prod_{e \in E(G)} n_{e} z\right) \sum_{T}\left(\prod_{f \in T} \frac{1}{n_{f} z}\right) \\
& =z^{n(G)} \sum_{T} \prod_{e \notin T} n_{e} .
\end{aligned}
$$

(2) The case of $D_{s}(G)$.

In this case $w(e)=n_{e} z$ and $\nu(e)=1$. Hence,

$$
\begin{aligned}
\nabla_{D_{s}(G)}(z) & =\left.\left(\frac{z}{v^{-1}-v}\right) Q_{G}\left(\frac{v^{-1}-v}{z}, \frac{v^{-1}-v}{z}\right)\right|_{v=1} \\
& =\sum_{F \subset E(G)}\left(\prod_{f \in F} w(f)\right)\left(\frac{v^{-1}-v}{z}\right)^{k\langle F\rangle-1}\left(\frac{v^{-1}-v}{z}\right)^{n\langle F\rangle} \\
& =\sum_{T}\left(\prod_{f \in T} n_{f} z\right) \\
& =z^{r(G)} \sum_{T} \prod_{e \in T} n_{e} .
\end{aligned}
$$


It is natural that one may expect that the values of $\beta_{T}(v, z)=\frac{L(T)-M(T)}{N(T)}$ will be different, i.e. depending on the alternatingly oriented tangle $T$. In this sense the following result is a little surprising.

Lemma 4.7. For any alternatingly oriented tangle $T, \beta_{T}(v, z)=\frac{v^{-1}-v}{z}$.

Proof. From the proof of Lemma 4.1 we know that $L(T)=P_{D e(T)}$, where $D e(T)$ is the denominator of the alternatingly oriented tangle $T$. Enlightened by the proof of Lemma 4.1, we can see that for an alternatingly oriented tangle as shown in Figure 2 (right), we have the following decomposition:

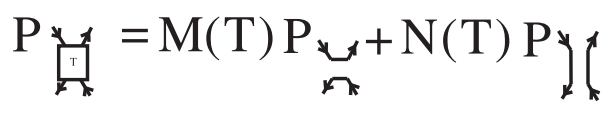

where $M(T)$ and $N(T)$ are Laurent polynomials in $v$ and $z$. Let $N u(T)$ be the numerator of the oriented tangle $T$. Then we have

$$
\begin{aligned}
& P_{D e(T)}=M(T) P_{\bigcirc}+N(T) P_{\bigcirc}, \\
& P_{N u(T)}=M(T) P_{\bigcirc}, N(T) P_{\bigcirc} .
\end{aligned}
$$

By solving the above two equations, we obtain

$$
\begin{aligned}
M(T) & =\frac{\frac{v^{-1}-v}{z} P_{N u(T)}-P_{D e(T)}}{\left(\frac{v^{-1}-v}{z}\right)^{2}-1}, \\
N(T) & =\frac{\frac{v^{-1}-v}{z} P_{D e(T)}-P_{N u(T)}}{\left(\frac{v^{-1}-v}{z}\right)^{2}-1} .
\end{aligned}
$$

Hence,

$$
\begin{aligned}
\beta_{T}(v, z) & =\frac{L(T)-M(T)}{N(T)} \\
& =\frac{\left(\left(\frac{v^{-1}-v}{z}\right)^{2}-1\right) P_{D e(T)}-\left(\frac{v^{-1}-v}{z} P_{N u(T)}-P_{D e(T)}\right)}{\frac{v^{-1}-v}{z} P_{D e(T)}-P_{N u(T)}} \\
& =\frac{v^{-1}-v}{z} .
\end{aligned}
$$

Thus, Theorem 4.2 becomes

Theorem 4.8. Let $G$ be a plane graph and $D(G)$ be its associated link diagram. Let $\delta=\frac{v^{-1}-v}{z}$. Then

$$
P_{D(G)}(v, z)=\delta^{-1}\left(\prod_{e \in E(G)} \mu(e)\right) Q_{G}(\delta, \delta),
$$

where $\mu(e)=\frac{\delta P_{N u\left(T_{e}\right)}-P_{D e\left(T_{e}\right)}}{\delta^{2}-1}$ and the weight $w(e)$ of edge e of $G$ is $\frac{\delta P_{D e\left(T_{e}\right)}-P_{N u\left(T_{e}\right)}}{\delta P_{N u\left(T_{e}\right)}-P_{D e\left(T_{e}\right)}}$.

Remark 4.9. Let $D_{u}(G)$ be the uniformly oriented link obtained from $G$ by replacing every edge by a fixed alternatingly oriented tangle $T$. Then the weight $w(e)$ in Theorem 4.8 will be the same for all $e$. In this case, by Lemma 2.2 in [13, the computation of the Homfly polynomial of $D_{u}(G)$ can be further reduced to computing 
the Tutte polynomial of the graph $G$. In particular, the principal Proposition 1 in [11 can thus be obtained. Note that the Maple software has a function called the TuttePolynomial in the GraphTheory package, which can be used to calculate the Tutte polynomial of small graphs.

Remark 4.10. The colored Tutte polynomial [3] and the chain polynomial [20] are, at least to some extent, both versions of edge weighted Tutte polynomials. It is not difficult to convert the computation of dichromatic polynomial of weighted graphs to those of the Tutte polynomial of colored graphs and the chain polynomial of labeled graphs.

\section{The Jaeger and Traldi Results}

In this section, we explain explicitly how Jaeger's and Traldi's results come from our results. The definition of the Homfly polynomial has several different forms, although all these forms are equivalent. We take the 2-variable form in this paper, while F. Jaeger and L. Traldi in their papers take the 3 -variable form.

Let $G$ be a plane graph and let $D_{1}(G)$ be Traldi's oriented link diagram obtained from $G$ by replacing each edge by one of four types of oriented clasps as shown in Figure 1. Let $a, b, c$ and $d$ be the number of edges of $G$ which are replaced by the first, second, third and fourth oriented clasps (from top to bottom), respectively. Note that the first and the second clasps are vertical integer tangles [2] and [-2], and the third and the fourth clasps are horizontal integer tangles $\overline{[2]}$ and $\overline{[-2]}$. According to Theorem 4.3, we obtain the 2-variable form of Traldi's Theorem 5 in [22].

Theorem 5.1. Let $G$ be a plane graph. Let $D_{1}(G)$ be Traldi's oriented link diagram. Let the weight of the edge replaced by the first, second, third and fourth oriented clasps be $\frac{v}{z},-\frac{1}{v z}, \frac{z}{v}$ and $-v z$, respectively. Then

$$
P_{D_{1}(G)}(v, z)=\left(\frac{z}{v^{-1}-v}\right)(v z)^{a}\left(-\frac{z}{v}\right)^{b} v^{2 c-2 d} Q_{G}\left(\frac{v^{-1}-v}{z}, \frac{v^{-1}-v}{z}\right) .
$$

Let $G$ be a connected plane graph with vertex set $V(G)$ and edge set $E(G)$, and let $D^{1}(G)$ be Jaeger's oriented link obtained from $G$ by replacing each edge by the first type of clasp. Using Theorem 5.1] and Lemma 2.2 in [13] we obtain the 2-variable form of Proposition 1 in [1]:

$$
\begin{aligned}
P_{D^{1}(G)}(v, z) & =\left(\frac{z}{v^{-1}-v}\right)(v z)^{|E(G)|} Q_{G}\left(\frac{v^{-1}-v}{z}, \frac{v^{-1}-v}{z}\right) \\
& =\left(\frac{z}{v^{-1}-v}\right)(v z)^{|E(G)|}\left(\frac{v}{z}\right)|V(G)|-1 \\
& =\left(\frac{v}{z}\right)^{|V(G)|-1}(v z)^{|E(G)|} T_{G}\left(v^{-2}, 1+\frac{1-v^{2}}{z^{2}}\right) .
\end{aligned}
$$




\section{ACKNOWLEDGEMENTS}

This work was supported by grants from the National Natural Science Foundation of China (No. 10831001) and the Fundamental Research Funds for the Central Universities (No. 2010121007). The first author thanks Professor L. Traldi for some helpful comments. We thank the referee for useful advice.

\section{REFERENCES}

1. J. W. Alexander, Topological invariants of knots and links, Tran. Amer. Math. Soc. 30 (1928), 275-306. MR1501429

2. B. Bollobaś, Modern Graph Theory, Springer, 1998. MR1633290(99h:05001)

3. B. Bollobás, O. Riordan, A Tutte polynomial for colored graphs, Combin. Probab. Comput. 8 (1999), 45-93. MR1684623 (2000f:05033)

4. T. Brylawski, A combinatorial model for series-parallel networks, Trans. Amer. Math. Soc. 154 (1971), 1-22. MR0288039(44:5237)

5. J. H. Conway, An enumeration of knots and links, and some of their algebraic properties, Comput. Problems in Abstract Algebra, Pergamon Press, New York (1970), 329-358. MR 0258014 $(41: 2661)$

6. P. G. Cromwell, Knots and Links, Cambridge University Press, 2004. MR 2107964 (2005k:57011)

7. Y. Diao, C. Ernst, U. Zieglerc, Jones polynomial of knots formed by repeated tangle replacement operations, Toplogy Appl. 156 (2009), 2226-2239. MR2544132 (2010k:57025)

8. Y. Diao, G. Hetyei, K. Hinson, Tutte polynomials of tensor products of signed graphs and their applications in knot theory, J. Knot Theory Ramification 18 (2009), 561-590. MR 2527677 (2010c:57010)

9. S. Eliahou, L. H. Kauffman, M. B. Thistlethwaite, Infinite families of links with trivial Jones polynomial, Topology 42, no. 1 (2003), 155-169. MR1928648 (2003g:57015)

10. P. Freyd, D. Yetter, J. Hoste, W. B. R. Lickorish, K. Millett, A. Ocneanu, A new polynomial invariant of knots and links, Bull. Amer. Math. Soc. (N.S.) 12 (1985), 239-246. MR776477 (86e:57007)

11. F. Jaeger, Tutte polynomials and link polynomials, Proc. Amer. Math. Soc. 103 (1988), 647654. MR943099 (89i:57004)

12. X. Jin, F. Zhang, F. Dong, E. G. Tay, Zeros of the Jones polynomial are dense in the complex plane, Electron. J. Comb. 17, no. 1 (2010) R94. MR2661397

13. X. Jin, F. Zhang, The Homfly polynomial for even polyhedral links, MATCH Commun. Math. Comput. Chem. 63 (2010), 657-677. MR2666626 (2011c:92100)

14. V. F. R. Jones, A polynomial invariant for knots via Von Neumann algebras, Bull. Amer. Math. Soc. (N.S.) 12 (1985), 103-111. MR766964 (86e:57006)

15. L. H. Kauffman, A Tutte polynomial for signed graphs, Discrete Appl. Math. 25 (1989), 105-127. MR.1031266 (91c:05082)

16. S. Liu, X. Cheng, H. Zhang, W. Qiu, The architecture of polyhedral links and their HOMFLY polynomials, J. Math. Chem. 48, no. 2 (2010), 439-456. MR.2665344

17. K. Luse, Y. Rong, Examples of knots with the same polynomials, J. Knot Theory Ramification 15, no. 6 (2006), 749-759. MR2253833(2007f:57019)

18. J. G. Oxley, D. J. A. Welsh, Tutte polynomials computable in polynomial time, Discrete Math. 109 (1992), 185-192. MR1192381 (94e:05076)

19. J. H. Przytycki, P. Traczyk, Invariants of links of Conway type, Kobe J. Math. 4 (1987), 115-139. MR945888 (89h:57006)

20. R. C. Read, E. G. Whitehead Jr., Chromatic polynomials of homeomorphism classes of graphs, Discrete Math. 204 (1999), 337-356. MR1691877 (2000b:05059)

21. W. Schwärzler, D. J. A. Welsh, Knots, matroids and the Ising model, Math. Proc. Cambridge Philos. Soc. 113, no. 1 (1993), 107-139. MR1188822 (94c:57019)

22. L. Traldi, A dichromatic polynomial for weighted graphs and link polynomials, Proc. Amer. Math. Soc. 106 (1989), 279-286. MR 955462 (90a:57013)

23. L. Traldi, Parallel connections and colored Tutte polynomials, Discrete Math. 290 (2005), 291-299. MR2123398 (2005j:05033) 
24. W. T. Tutte, A contribution to the theory of chromatic polynomials, Canad. J. Math. 6 (1954), 80-91. MR0061366 (15:814c)

25. L. Waston, Any tangle extends to non-mutant knots with the same Jones polynomials, J. Knot Theory Ramification 15, no. 9 (2006), 1153-1162. MR.2287438 (2007k:57028)

26. D. R. Woodall, Tutte polynomial expansions for 2-separable graphs, Discrete Math. 247 (2002), 201-213. MR 1893028 (2002m:05094)

School of Mathematical Sciences, Xiamen University, Xiamen, Fujian 361005, PeoPLE'S REPUBlic OF CHINA

E-mail address: xajin@xmu.edu.cn 